\title{
Identification of Illustrators
}

\author{
Fadime Sener, Nermin Samet, and Pinar Duygulu Sahin \\ Computer Engineering Department, Bilkent University, Ankara, Turkey
}

\begin{abstract}
This paper is motivated by a book in which artists and illustrators from all over the world offer their personal interpretations of the declaration of human rights in pictures [1]. It was enthusiastic for a young reader to see an illustration of an artist that he already knows from his books. The characters were different, the topic was irrelevant, but still it was easy to identify the illustrators based on the style of the illustration. Inspired by the human's ability to identify illustrators, in this study we propose a method that can automatically learn to distinguish illustrations of different illustrators using computer vision techniques.
\end{abstract}

\section{Introduction}

With the increasing number of digital images of artwork that becomes available such as through Google Art Project 1, cross-disciplinary collaboration between art historians and computer scientists becomes more desirable.

Attempts in applying image processing and computer vision techniques to assist art scholars have shown good performance for analysis of perspective, and illumination [2]. Recently, machine learning techniques have been applied for classification of paintings, artists and styles [3 11].

Identification of an artist or an art style is important to detect replications or followers. Vincent van Gogh's paintings are identified through brushstrokes using wavelet transform based features 12]. The roots of Portuguese Tile Art are traced in [13, 14] based on visual similarities. In [15], a new shape descriptor is used to identify Mayan hieroglyphs.

Motivated by the studies in identification of painters, in this study we address another challenge and aim to identify the artistic works of illustrators. Rather than focusing on specific representations which may only work for some limited artistic works, we analyze the illustrations through advanced and general descriptors which are applied successfully on other computer vision problems. Our experiments on four artists illustrating children books show that successful performances can be obtained in identification of illustrators.

In the following, first the data collection will be introduced followed by the presentation of the descriptors. We then describe the details of our classification method. Finally, detailed experiments will be presented and discussed.

\section{Data Collection}

In this study, we focus on artists illustrating children books. 11] contains 30 articles of declarations of human rights in pictures collectively illustrated by

\footnotetext{
1 http://www.googleartproject.com/
} 
well-known artists. For three illustrators contributed to this book, namely Korky Paul, Axel Scheffler and Debi Gliori (see Figure 1), we were able to collect sufficient number of images either from the Internet or through scanning books. In addition to these images, we also included the illustrations of Dr. Seuss to construct a data collection.

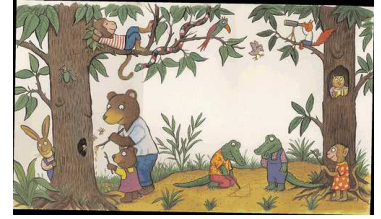

(a)

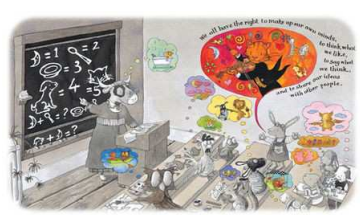

(b)

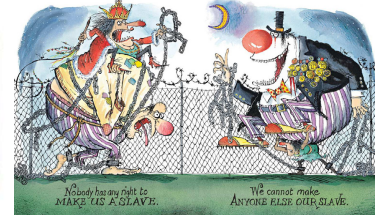

(c)

Fig. 1. Illustrations of (a) Axel Scheffler, (b) Debi Gliori and (c) Korky Paul in [1]

In our dataset we have 248 illustrations of Axel Scheffler, 243 illustrations of Debi Gliori, 249 illustrations of Korky Paul and 234 illustrations of Dr. Seuss. Figure 2 represents some example illustrations from the dataset.
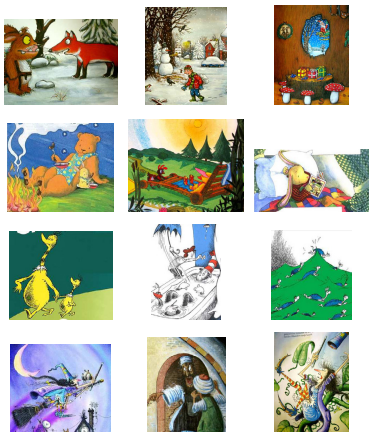
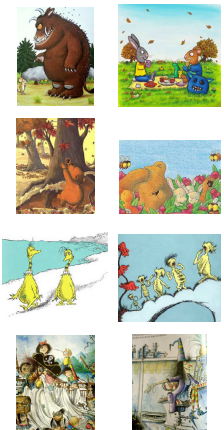
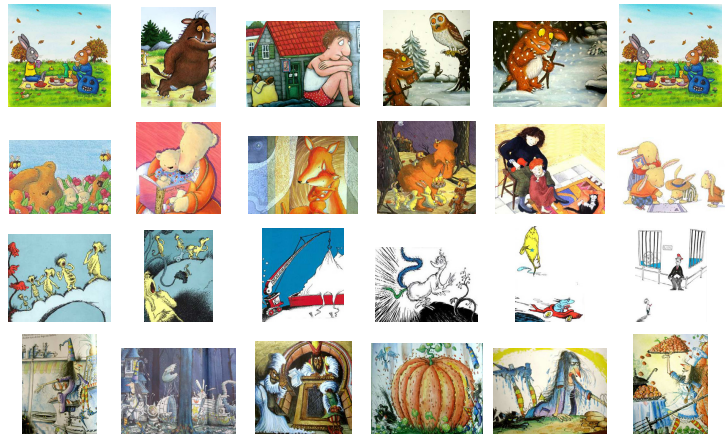

Fig. 2. Samples from Axel Scheffler ( $1^{\text {st }}$ row $)$, Debi Gliori $\left(2^{\text {nd }}\right.$ row $)$, Dr. Seuss $\left(3^{\text {rd }}\right.$ row) and Korky Paul ( $4^{\text {th }}$ row)

\section{Descriptors}

Color is an important property of illustrations for most of the artists: some artists prefer to use multiple colors while the others use less number of pure colors (see Figure 2). Based on this idea, as our first feature we choose to use $4 \times 4 \times 4$ bin RGB histograms. However, as it will be shown with the experiments, the performance of the color features are not sufficiently good; therefore more advanced features are studied. Namely, GIST [16], HOG [17], Dense SIFT [18] and Color Dense SIFT [18] features are extracted from each illustration. We generated GIST 
features for each illustration by computing with orientation scale 8 and 4 blocks. SIFT features are densely extracted from illustrations and then a codebook is generated for Bag-of-words [19] representation using k-means clustering. Color Dense SIFT is similar except it also contains color information.

\section{Classification}

Support Vector Machines are used for classification. In particular LIBSVM library [20] is used for SVM classification. We use one versus all approach for training. That is, to prepare the training set for a class, we provide the negative samples from all other classes. We labeled the training and test sets manually. A test example is fed into multiple classifiers and it is assigned to the class with the highest confidence value. Several different kernels were used for each set of features, including chi-square kernel, linear kernel, histogram intersection kernel, Radial Basis Function kernel and Hellinger's kernel.

\section{$5 \quad$ Experiments}

In the following, we will first provide detailed experimental evaluations to understand the effect of selected descriptors and classification methods in classifying illustrators. Then, focusing on Dr. Seuss we will present the results in separation of the original work from the works of followers.

\subsection{Evaluation of Descriptors and Classification Methods}

We first evaluate the performance of the descriptors on illustrators identification. In Figure 3, Figure 4 and Figure 5 we show the first 15 illustrations that have the highest confidence scores for the classifiers corresponding to four different artists for color histogram, GIST and HOG features respectively.

We can come up with some conclusions from these figures that are aligned with the humans' observations about the style of the illustrators. Dr. Seuss use a small range of characteristic colors. Most of Axel Scheffler illustrations have forest background so that these images have some constant colors. Korky Paul also has special background styles in terms of colors. These are represented with the performance of the color histogram feature. Compared to the other illustrators Debi Gliori's illustrations are less distinguishable with color. On the other hand GIST feature is more successful for Debi Gliori. HOG feature is failed for Debi Gliori and Korky Paul but it is successfull for Dr. Seuss and Axel Scheffler where the contours are more obvious.

Besides these three features, we also experimented BoW Dense SIFT and BoW Color Dense SIFT. These are the features obtained by extracting dense salient points, representing them by SIFT descriptors, and using k-means clustering to obtain bags of words. Both of these BoW SIFT based features show better performances compared to the others: The first 15 images were all correct for 


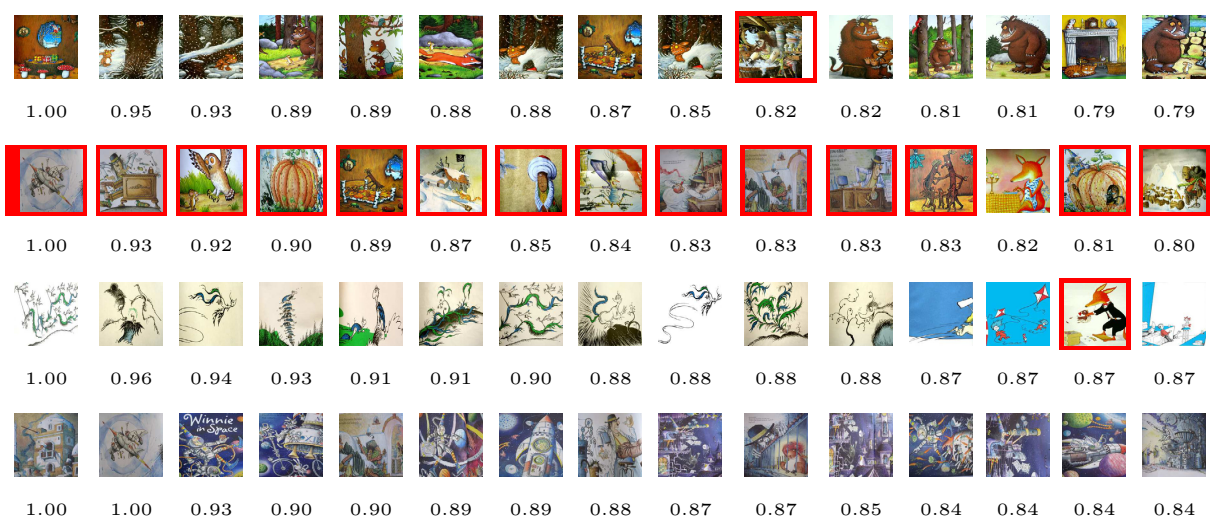

Fig. 3. Results of color histogram feature. Axel Scheffler ( $1^{\text {st }}$ row $)$, Debi Gliori $\left(2^{\text {nd }}\right.$ row), Dr. Seuss ( $3^{\text {rd }}$ row) and Korky Paul ( $4^{\text {th }}$ row). The numbers show the confidence values. Images in red boxes are the wrong results.

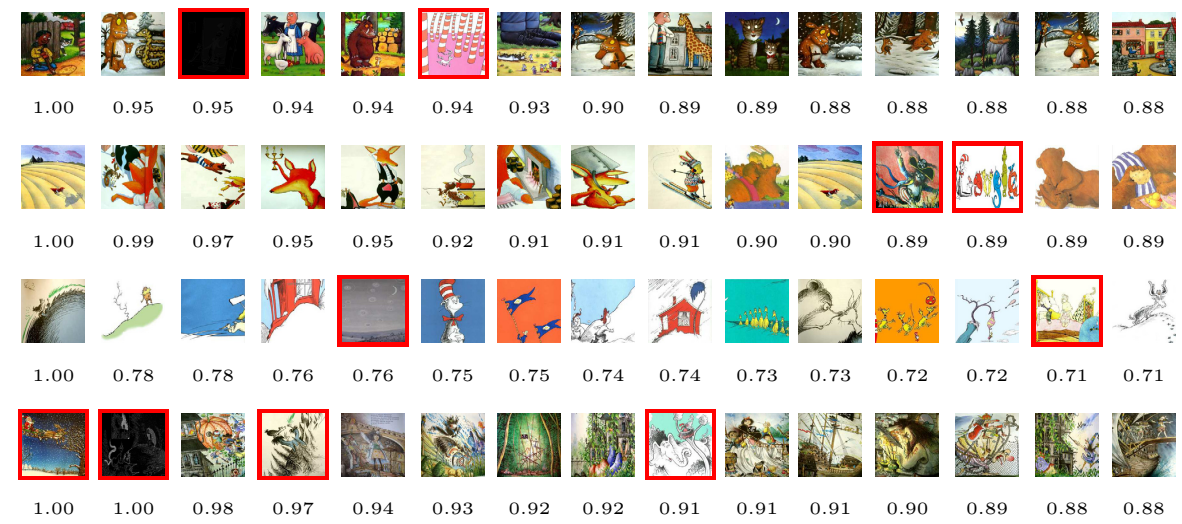

Fig. 4. Results of GIST feature. Axel Scheffler (1 ${ }^{\text {st }}$ row), Debi Gliori ( $2^{\text {nd }}$ row), Dr. Seuss $\left(3^{\text {rd }}\right.$ row $)$ and Korky Paul ( $4^{\text {th }}$ row $)$. The numbers show the confidence values. Images in red boxes are the wrong results.

all illustrators. As we observed through looking at the clusters, the reasons for the good performances is that the visual words (clusters) correspond to stylistic elements in the illustrations: such as the big eyes in Axel Scheffler or stars in Debi Gliori illustrations. That is, we were able to capture the important characteristics of the illustrators without any human intervention or without any specific training.

Figure 6 represents Precision-Recall curves for each illustrator for all the features experimented. As can be observed from these figures, compared to BoW Dense SIFT feature, BoW Color Dense SIFT has better performance in terms of average precision. Among all features, BoW is more capable to discriminate illustrations. Additionally when we use color SIFT which include color information we get the highest performance. 


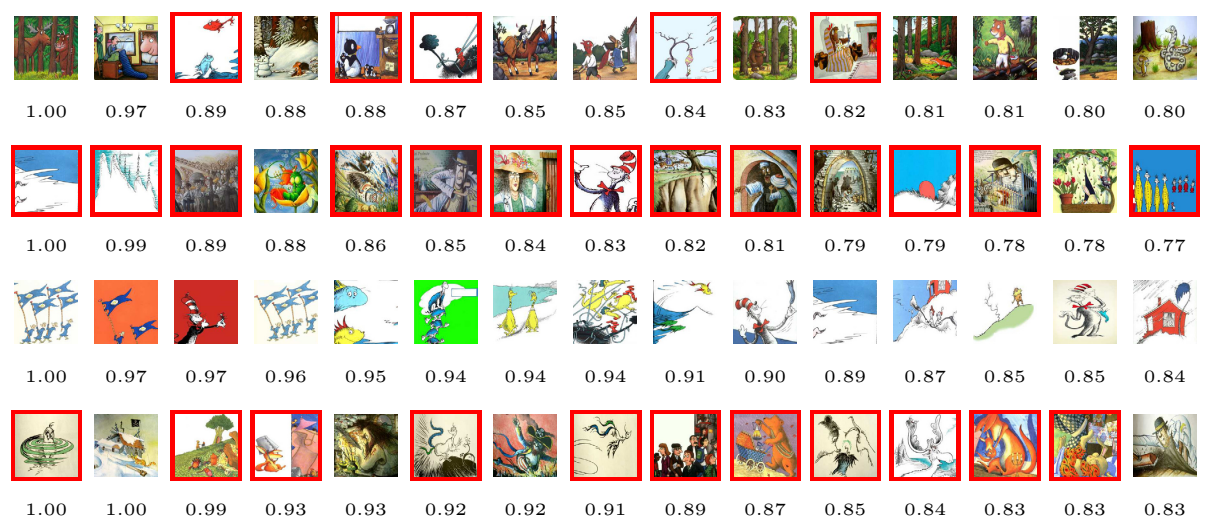

Fig. 5. Results of HOG feature. Axel Scheffler ( $1^{\text {st }}$ row), Debi Gliori ( $2^{\text {nd }}$ row), Dr. Seuss $\left(3^{\text {rd }}\right.$ row $)$ and Korky Paul $\left(4^{\text {th }}\right.$ row $)$. The numbers show the confidence values. Images in red boxes are the wrong results.

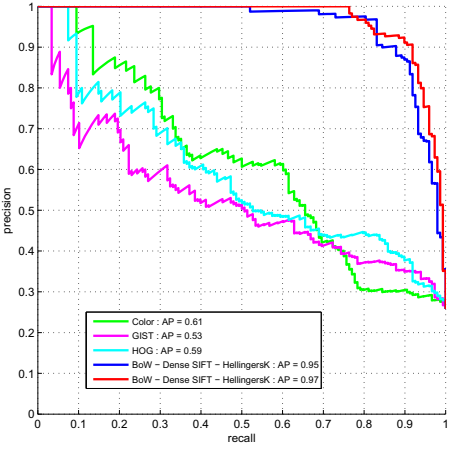

(a)

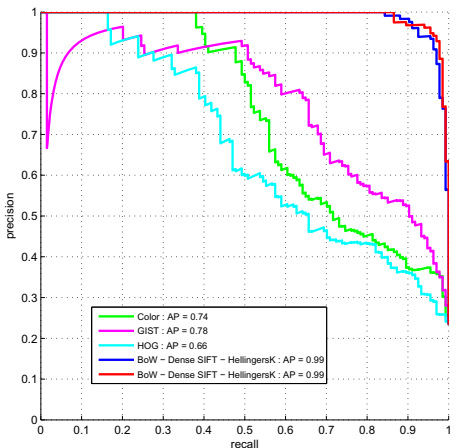

(c)

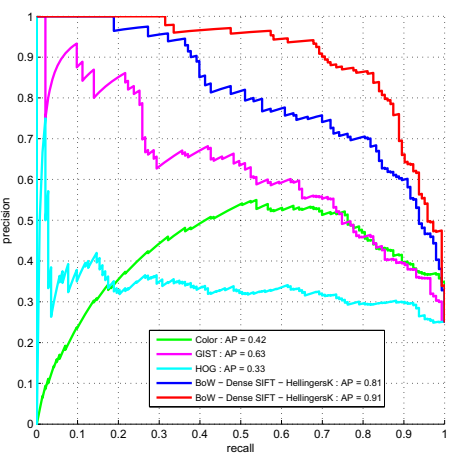

(b)

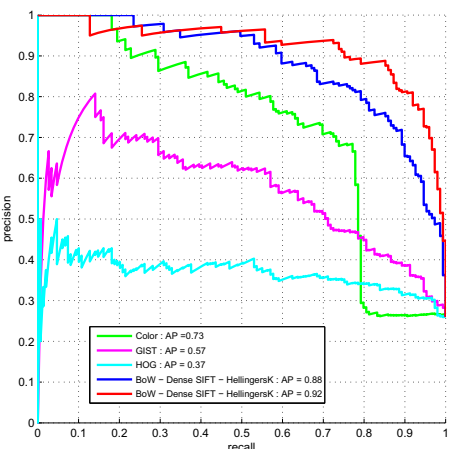

(d)

Fig. 6. Precision-Recall curves of features for (a) Axel Scheffler, (b) Debi Gliori, (c) Dr. Seuss and (d) Korky Paul 
For classification, we use one versus all approach. Among different kernels experimented, Hellinger's kernel has the best performance and it has less computation time than others. Over our baseline where we use linear SVM, using Hellinger's kernel did not have any effect on color histogram, HOG and GIST features, but it increased the performances of BoW SIFT based approaches. Overall performances are given in Figure 7 for different size of training data. Figure 8 presents the results on each illustrator separately.

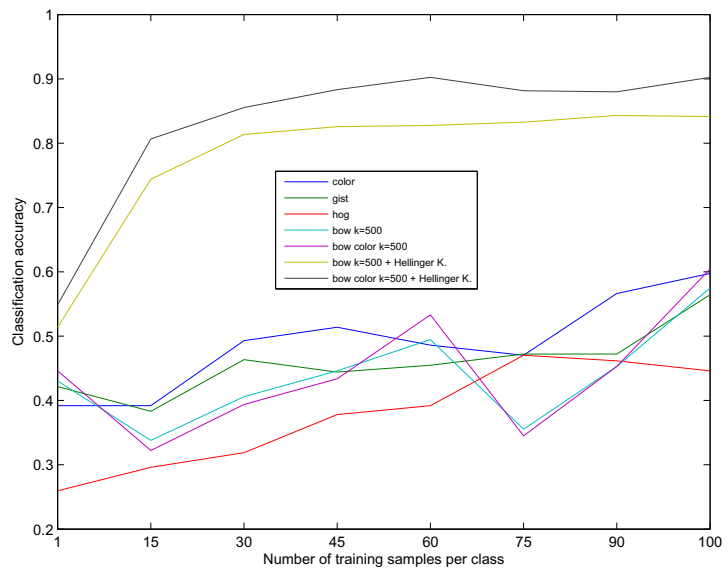

Fig. 7. Overall classification performances for different features. BoW Color SIFT feature with Hellinger's kernel outperforms others.

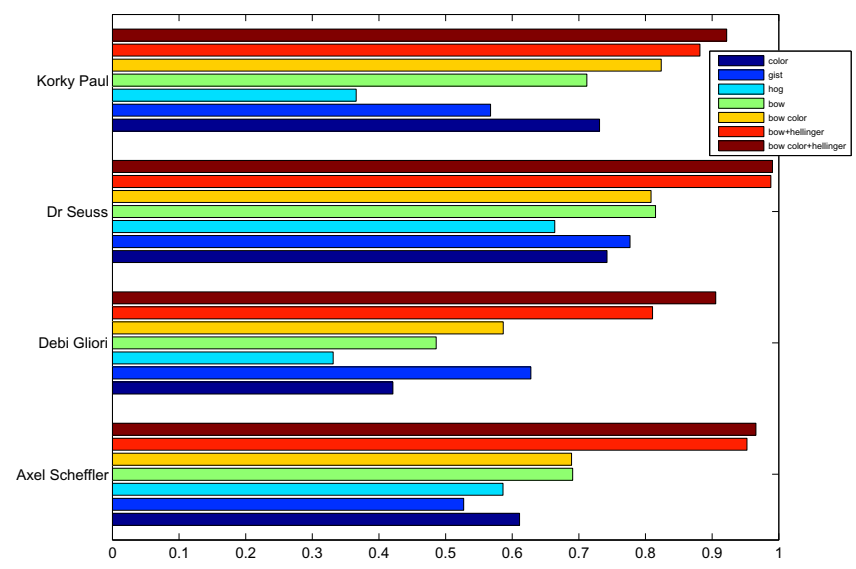

Fig. 8. Classification performances for each illustrator. Among all others BoW Color with Hellingers kernel has the best performance for each illustrator.

Since BoW Color Dense SIFT has better performances, we focused on this feature and evaluated the effect of vocabulary size (see Table1). We obtain best results with $\mathrm{k}=1000$. 
Table 1. Vocabulary size performances

\begin{tabular}{cc}
\hline \hline $\mathrm{k}:$ codeword size & Test data performance \\
\hline $\mathrm{k}=500$ & 0.86 \\
$\mathrm{k}=600$ & 0.87 \\
$\mathrm{k}=700$ & 0.88 \\
$\mathrm{k}=800$ & 0.88 \\
$\mathrm{k}=900$ & 0.90 \\
$\mathrm{k}=1000$ & 0.91 \\
\hline
\end{tabular}

These results were obtained with random sampling of training and test data where 100 samples are used for training and the rest is used for testing. In order to test the performance of our methods on different randomly selected samples, we performed 10-fold cross validation (see Figure 9). The results show that BoW Color Dense SIFT has the least variance.

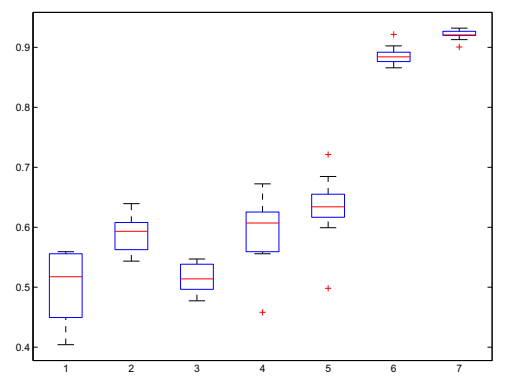

Fig. 9. Results for 10 fold cross validation: Color, GIST, HOG, BoW-SIFT, BoWColor SIFT, BoW-SIFT with Hellinger's kernel, BoW- Color SIFT with Hellinger's kernel respectively

\subsection{Identification of Followers}

Dr. Seuss's style is adapted in a series of books by different illustrators. In the first look, it is difficult to distinguish the originals from the followers. Motivated with this challenge, we perform additional experiments in order to separate original Dr. Seuss's illustrations from the others. We obtain $91 \%$ accuracy with binary classification. In Figure10, we show some examples of the followers which are confused as the original Dr. Seuss illustrations.

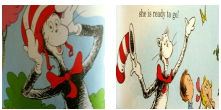

20

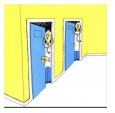

64

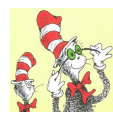

71

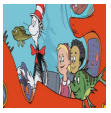

87

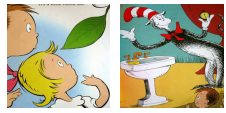

91

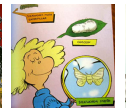

104

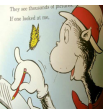

112

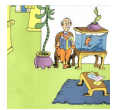

114

Fig. 10. Illustrations of the followers which are confused as the original Dr. Seuss works with their ranking indexes 


\section{Summary and Discussions}

In this study, we address the challenge of identifying illustrators. Our experiments show that, even with general descriptors which are not specific to any artistic style analysis, it is possible to identify the works of different illustrators. For the examples adapted from [1], our classifiers were successful in identifying the correct illustrators. This shows that even within different themes or with different characters, the style characteristics of illustrators can be captured with the proposed method. Our experiments on distinguishing the originals from the followers with high performances also suggest that the proposed method can be applied for other purposes, such as for detecting unauthorized copies.

In the future, we plan to extend the set of illustrators and also to focus on more advanced descriptors such as for capturing the styles of artists in illustrating the faces, eyes, etc.

Acknowledgments. We would like to thank Ardic for inspiring us and for his help in creating the dataset.

\section{References}

1. We Are All Born Free: The Universal Declaration of Human Rights in Pictures. Frances Lincoln (2008)

2. Stork, D.: Computer image analysis of paintings and drawings: an introduction to the literature. In: The 1st International Workshop on Image Processing for Artist Identification, Amsterdam, The Netherlands (2008)

3. Sablatnig, R., Kammerer, P., Zolda, E.: Hierarchical classification of paintings using face- and brush stroke models. In: 14th International Conference on Pattern Recognition, vol. 1, pp. 172-174 (1998)

4. Kroner, S., Lattner, A.: Authentication of free hand drawings by pattern recognition methods. In: 14th International Conference on Pattern Recognition, vol. 1, pp. 462-464 (1998)

5. Keren, D.: Painter identification using local features and naive bayes. In: 16th International Conference on Pattern Recognition, vol. 2, pp. 474-477 (2002)

6. Icoglu, O., Gunsel, B., Sariel, S.: Classification and indexing of paintings based on art movements. In: Proceedings of European Signal Processing Conference (EUSIPCO), Vienna, Austria, pp. 749-752 (2004)

7. Lombardi, T.: The Classification of Style in Painting: Computational Approaches to Artistic Style. VDM Verlag (2008)

8. Legrand, A., Vurpillot, V., Tremeau, A., Schettini, R.: Automatic color patch selection for painting identification. In: 4th European Conference on Colour in Graphics, Imaging, and Vision (CGIV), pp. 300-303 (2008)

9. Zujovic, J., Gandy, L., Friedman, S., Pardo, B., Pappas, T.N.: Classifying paintings by artistic genre: An analysis of features and classifiers. In: Proceedings of IEEE International Workshop on Multimedia Signal Processing (MMSP), Rio de Janeiro, Brazil (2009) 
10. Antaresti, T., Arymurthy, A.M.: Image feature extraction and recognition of abstractionism and realism style of indonesian paintings. In: Proceedings of the 2010 Second International Conference on Advances in Computing, Control, and Telecommunication Technologies (ACT 2010), Washington, DC, USA, pp. 149$152(2010)$

11. Blessing, A., Wen, K.: Using machine learning for identification of art paintings. Technical report, Stanford University (2010)

12. Johnson, C.R., Hendriks, J.E., Berezhnoy, I.J., Brevdo, E., Hughes, S., Daubechies, I., Li, J., Postma, E., Wang, J.Z.: Image processing for artist identification. IEEE Signal Processing Magazine, 37-48 (2008)

13. Cabral, R., Costeira, J.P., la Torre, F.D., Bernardino, A., Carneiro, G.: Time and order estimation of paintings based on visual features and expert priors. In: Proc. of the Conference on Computer Vision and Analysis of Images of Art II, San Francisco, USA (2011)

14. da Silva, N.P., Marques, M., Carneiro, G., Costeira, J.P.: Explaining scene composition using kinematic chains of humans: application to portuguese tiles history. In: Proc. of the Conference on Computer Vision and Analysis of Images of Art II, San Francisco, USA (2011)

15. Roman-Rangel, E., Pallan, C., Odobez, J.M., Gatica-Perez, D.: Analyzing ancient maya glyph collections with contextual shape descriptors. Int. Journal of Computer Vision, Special Issue on e-Heritage 94, 101-117 (2011)

16. Oliva, A., Torralba, A.: Modeling the shape of the scene: A holistic representation of the spatial envelope. International Journal of Computer Vision 42, 145-175 (2001)

17. Dalal, N., Triggs, B.: Histograms of oriented gradients for human detection. In: Schmid, C., Soatto, S., Tomasi, C. (eds.) International Conference on Computer Vision \& Pattern Recognition, vol. 2, INRIA Rhône-Alpes, ZIRST-655, av. de l'Europe, Montbonnot-38334, pp. 886-893 (2005)

18. Lowe, D.G.: Distinctive image features from scale-invariant keypoints. Int. J. Comput. Vision 60, 91-110 (2004)

19. Sivic, J., Russell, B.C., Efros, A.A., Zisserman, A., Freeman, W.T.: Discovering object categories in image collections. In: Proceedings of the International Conference on Computer Vision (2005)

20. Chang, C.C., Lin, C.J.: LIBSVM: A library for support vector machines. ACM Transactions on Intelligent Systems and Technology 2, 27:1-27:27 (2011), Software available at http://www.csie.ntu.edu.tw/ cjlin/libsvm 